\title{
Surface Science Approaches to Molecular Nanostructures
}

\author{
Matthias Treier ${ }^{a}$ and Roman Fasel ${ }^{\star a b}$
}

\begin{abstract}
The self-assembly of large organic adsorbates on solid surfaces is driven by subtle energy balances between adsorbate-adsorbate and adsorbate-substrate interactions. Understanding these interactions is a key step towards the rational design and large-scale production of ordered, two-dimensional organic nanostructures which may find applications in (opto)electronic devices, sensors and surface catalysts. Due to the reduced dimensionality at surfaces, new phenomena arise which can only be understood by combining both experimental and theoretical methods and knowledge from chemistry and physics. In this short review, we illustrate the richness of surface chemical phenomena at the hand of four case studies which are selected to highlight the potential as well as the current limitations in controlling molecular self-assembly at surfaces.
\end{abstract}

Keywords: Polycyclic aromatic hydrocarbons · Polyimide · Scanning tunneling microscopy · Self-assembly · Supramolecular architectures

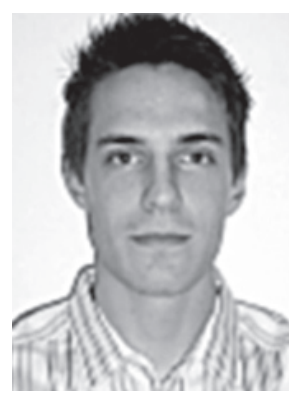

Matthias Treier studied physics at the Ecole Polytechnique Fédérale de Lausanne and at the University of Nottingham. He received his MSc from EPFL in 2005 and recently finished his $\mathrm{PhD}$ at the University of Zürich. He is currently a post-doc in the nanotech@surfaces Laboratory at Empa. His main research interests are in the field of surface-supported organic nanostructures, including aspects of organic, polymer and supramolecular chemistry.

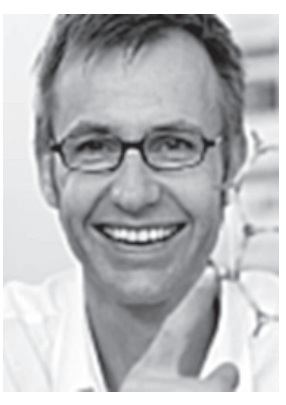

Roman Fasel received his $\mathrm{PhD}$ in physics in 1996 from the University of Fribourg and joined Empa after post-docs at $\mathrm{La}$ Trobe University (Melbourne) and the Fritz-Haber-Institute (Berlin). $\mathrm{He}$ is a group leader in the nanotech@surfaces Laboratory of Empa, and since 2008 Tit. Professor at the Department of Chemistry and Biochemistry of the University of Bern. His group covers a wide range of activities from fundamental to applied research in the field of nanosized organic and inorganic structures at surfaces.

\section{Introduction}

Research on nanoscale objects and phenomena has seen an enormous increase over the past decades, motivated by a seemingly unlimited range of potential applications. Along with the discoveries of new phenomena arising from quantum size effects (that become important on the nanoscale) goes a removal of the boundaries between traditionally independent areas of science such as chemistry and physics. Surface science can be regarded as one of the meeting points between the two disciplines, with surface phenomena being relevant to areas such as solid state physics, crystallography, catalysis or synthetic and physical chemistry. The reduced dimensionality at surfaces, the resulting restraints on the degrees of freedom of adsorbates and the specific electronic structure have direct consequences on chemical interactions and reactions, such that 'chemistry in two dimensions' can vary considerably from more 'traditional' chemical environments (see e.g. refs [1-4]).

Besides the fundamental interest in surface chemical phenomena, interest in organic nanostructures is fuelled by possible applications in future electronic and optoelectronic devices where functional units might ultimately be comprised of single molecules. ${ }^{[5]}$ While proof-ofprinciple experiments have successfully shown that single molecules might be used as switches ${ }^{[6,7]}$ or transistors, ${ }^{[8,9]}$ the technological implementation of such devices will require the ability to place them at well-defined sites on a substrate and to interlink them into functional circuits. Since sequential positioning of individual molecules is highly inefficient and hence technologically unfeasible the use of guided self-assembly[10] is intensively explored. The ultimate goal is to gain the ability to position individual molecules - bearing a pre-designed, chemically tailored functionality - in a controlled manner on well-defined sites with control over conformation and orientation, and to interlink them into functional devices.

Apart from the potential use of single molecules, thin films of organic materials have already found application in electronic devices such as thin film transistors, [11] photosensors/solar cells ${ }^{[12]}$ and organic light emitting diodes. ${ }^{[13]}$ In order to improve the properties of such devices and to extend their applicability, it is of central importance to obtain both highly ordered and structured organic thin films and nanostructures, whose properties can be tuned by chemical synthesis, geometric shape and dimension of its subunits and the electronic coupling to the substrate.

Freiestrasse 3

$\mathrm{CH}-3012$ Bern 
This review shall give an overview on recent advances in this strongly interdisciplinary field of research where surface scientists, synthetic chemists and specialists in theory and modeling closely collaborate with the goal of ultimately establishing generally applicable principles for the fabrication and use of surface-supported supramolecular device components. Rather than attempting to cover this very lively field in all its dimensions, we will focus on recent surface science approaches towards the bottom-up fabrication of surface-supported organic nanostructures based on selected examples from our own laboratory. We will, in particular, neither touch on low-dimensional coordination architectures and hydrogenbonded supramolecular assemblies which have recently been reviewed by Barth, ${ }^{[14]}$ nor on supramolecular self-assembly at the liquid-solid interface. ${ }^{[15]}$ The examples presented in the following evidence a subtle balance of interactions taking place in supramolecular pattern formation on surfaces: Apart from intermolecular interactions, molecule-surface interactions clearly play an important role on their own, and we will see that they may be exploited to control molecular self-assembly or to assist in the formation of surface-supported polymeric nanostructures. Detailed insight into these phenomena is gained from low-temperature scanning tunneling microscopy (STM) studies under highly controlled ultra-high vacuum (UHV) conditions on single-crystal metal surfaces.

\section{Site-selective Anchoring of Molecular Building Blocks}

Different types of lithographic techniques have been used to imprint structures on surfaces, surface-supported monolayers or (organic) thin films. However, these techniques are inherently limited with respect to the ultimate feature size that can be achieved. To overcome this limitation, naturally templated substrates such as vicinal surfaces ${ }^{[16]}$ or strain-relief networks, ${ }^{[17]}$ exhibiting true nanoscale templating, can be used. Such templates have for example been used for the creation of regular metallic nanodot arrays. ${ }^{[18,19]}$

The vicinal Au(11 12 12) crystal (see inset in Fig. 1A) is naturally templated in one direction by a regular succession of steps and - along the direction of the step edges by the periodic change between $f c c$ and $h c p$ stacking areas which are separated by the socalled discommensuration lines (bright lines in Fig. 1A). This substrate is hence naturally templated in two dimensions with a rectangular unit cell of $5.8 \mathrm{~nm} \times 7.2 \mathrm{~nm}$.

We have recently shown ${ }^{[20]}$ that the organic semiconductor hexa-peri-hexabenzocoronene (HBC, Fig. 1B) preferentially
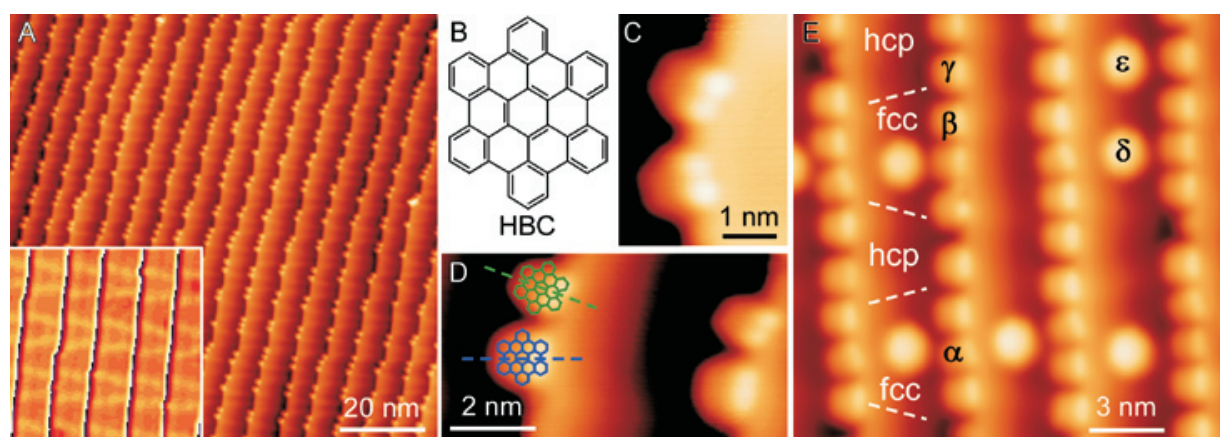

Fig. 1. (A) Large scale STM image of 0.1 monolayer (ML) HBC adsorbed on Au(11 12 12). The inset shows the bare template surface with maximum contrast applied to each terrace individually (25 nm $\times 25 \mathrm{~nm}$ ). (B) Chemical structure of HBC. (C) High resolution STM image of two kinkadsorbed HBC molecules. (D) STM image of kink adsorbed (upper, green) and step-adsorbed (lower, blue) HBC molecules. Dashed lines show the molecular axes, highlighting the rotation with respect to the step edge normal for kink-adsorbed HBC. (E) Hierarchy of adsorption sites for HBC on $\mathrm{Au}(111212)$ at about $0.2 \mathrm{ML}$ coverage (see text for details).

decorates kinks within $f c c$-stacking regions on randomly oriented steps on $\mathrm{Au}(111)$. As shown in Fig. 1A, HBC also selectively decorates kinks within $f c c$-stacking regions on the templated $\mathrm{Au}\left(\begin{array}{l}11 \\ 12\end{array} 12\right)$ crystal at low coverage, hence forming a regular array of subunits consisting of 1-3 HBC molecules, which are regularly spaced due to the nanostructured template surface. Characteristic of this adsorption are two protrusions centered over two of the peripheral benzene rings which appear to be lying on the upper step edge (Fig. 1C), suggesting that the molecules adsorb in a tilted way across the step edges. STM is however not able to clearly distinguish between electronic and geometric effects, such that the tilted adsorption geometry of $\mathrm{HBC}$ had to be proven experimentally by complementary X-ray photoelectron diffraction measurements. ${ }^{[21]} \mathrm{A}$ tilt angle of about $12^{\circ}$ of the aromatic board with respect to the $\mathrm{Au}(111)$ terraces has been determined experimentally and confirmed by an extensive density functional theory (DFT) analysis. ${ }^{[20]}$ A second characteristic feature of this adsorption configuration is a rotation of about $18^{\circ}$ of the molecular axis with respect to the step edge normal direction (Fig. 1D). While preferential decoration of surface defects is widely observed for most adsorbates, the tilted adsorption geometry across kinks is unusual, especially since adsorbates containing extended polycyclic aromatic units are generally expected to adsorb on coin metal substrates with their polyaromatic board parallel to the surface. ${ }^{[22]}$

A stepwise increase of the HBC coverage allows five energetically inequivalent adsorption sites on the $\mathrm{Au}\left(\begin{array}{lll}11 & 12 & 12\end{array}\right)$ substrate to be identification, as indicated in Fig. 1E. Adsorption across kinks is energetically favored (denoted as $\alpha$ in Fig. $1 \mathrm{E})$, followed by adsorption across straight step edges within $f c c$-stacking regions $(\beta)$. Once the step edges (straight and kinked) within the $f_{c c}$ regions are fully occupied, steps within the hcp regions are decorated $(\gamma)$. After full step decoration, subsequent adsorbates will decorate the $f c c$-stacking regions on the terraces $(\delta)$ in a way that only one HBC is found per $f c c$-region. At slightly higher coverage, single molecules will also decorate $h c p$-stacking regions $(\varepsilon)$ in the same way. This energetic hierarchy of adsorption sites nicely highlights the influence of both the co-ordination of the surface atoms (kinks and steps vs. terrace atoms) and their electronic structure ( $f c c$ vs. hcp stacking regions) on the interaction with adsorbates, which is of course also relevant for catalytic processes.

The same type of vicinal Au substrate can also be used for the formation of regularly spaced bimolecular ribbons and chains ${ }^{[23}$ or the formation of highly ordered arrays of $\mathrm{C}_{60}$ nanochains. [24] Fig. 2 shows that $\mathrm{C}_{60}$ chains consisting of 3-5 molecules are aligned along the step edges. As for HBC, $\mathrm{C}_{60}$ preferentially decorates $f c c$-stacking regions with the molecules sitting at the lower step edges. As shown by these examples, vicinal crystals can be used for the creation of highly ordered organic nanostructures, with the template surface periodicity being transferred to the organic superlattice.

Other substrates which have been successfully used as templates for organic nanostructures include a room-temperature stable nanohole array produced by sputtering the $\mathrm{Ag} / \mathrm{Pt}$ strain-relief network surface, ${ }^{[25]}$ the boron nitride 'nanomesh' monolayer on $\mathrm{Rh}(111),{ }^{[26]}$ partially nitrided $\mathrm{Cu}(001)^{[27]}$ or - with less long-range order of the resulting nanostructure array - the reconstructured $\mathrm{Au}(111)$ surface. ${ }^{[28]}$

\section{Aromatic Interactions in Two- dimensional Supramolecular Architectures}

The site-selective anchoring of a first adsorbate - as demonstrated in the preceding section and also shown for the HBC 


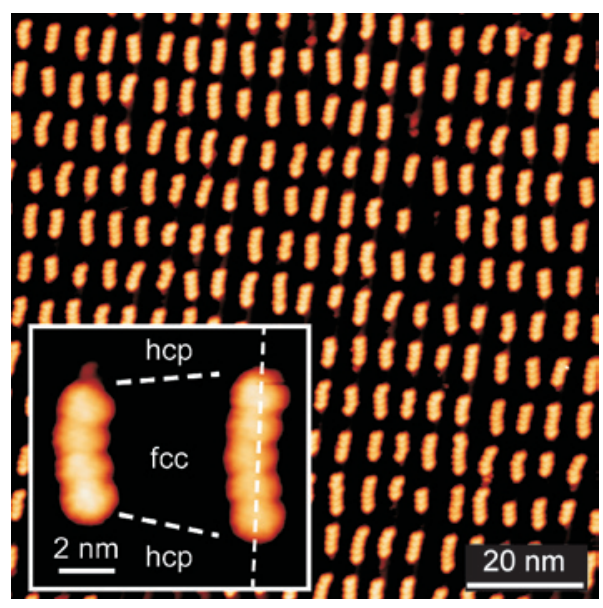

Fig. 2. STM image of a $C_{60}$ nanochain array on $\mathrm{Au}(1112$ 12). The inset shows a close-up of two chains consisting of four (left) and five (right) molecules.

derivate dodecamethyl-HBC[21] - might be used as a first step towards supramolecular architectures by using a specifically functionalized primary adsorbate. Secondary (ternary) co-adsorbates might then be connected to the initial anchors, thus allowing for the creation of a supramolecular system which can be grown at well-defined positions with respect to the substrate. Supramolecular architectures based on hydrogen bonding, , $14,23,29]$ metal co-ordination ${ }^{[14,30,31]}$ or a combination of the two ${ }^{[32]}$ have successfully been grown on single-crystal metal substrates before, with the ability to tune the networks through chemical functionalization. We have recently shown that a rational design of non-covalently bound supramolecular networks can also be achieved by the use of aromatic interactions, extending the 'library' of binding motifs - or synthons ${ }^{[33]}$ - for non-covalent two-dimensional (2D) supramolecular architectures by the class of aromatic interactions.

Aromatic stacking interactions are highly versatile and generally only show limited directionality[34] compared to other non-covalent interactions such as hydrogen bonds, ${ }^{[35]}$ which has hitherto limited their use in rationally designed 2D supramolecular architectures. Besides their relevance to the folding of large biomolecules and in crystal engineering, interest in aromatic stacking is also fuelled by the use of polyaromatic molecules as organic semiconductors in electronic devices where the use of polycyclic units appears to be a unifying design feature. ${ }^{[36,37]}$ In these devices, charge-carrier pathways are created due to the overlap between $\pi$-orbitals of nearest neighbor in face-to-face stacking geometries, so-called $\pi-\pi$ stacking.

While lateral aromatic interactions have been suggested to play a role in the selfassembly of organic adsorbates before, [38,39] there have been no prior studies on the possibility to rationally design supramolecular systems based on aromatic interactions. We have studied the adsorption behavior of the nonplanar polycyclic hydrocarbon hexacata-hexabenzoroconene (cHBC, inset in Fig. 3A) ${ }^{[40]}$ on $\mathrm{Cu}(111) .{ }^{[41]}$ Steric hindrance between neighboring hydrogen atoms in the bay regions distorts the aromatic board away from planarity. Due to this nonplanarity, molecules can partially interdigitate (see inset in Fig. 3A). At submonolayer coverage, cHBC predominantly forms a commensurate honeycomb superstructure on $\mathrm{Cu}(111)$ as shown in Fig. 3A. This network is stabilized by aromatic interactions between interdigitated self-complementary tetrahelicene units of nearest neighbors (see model in Fig. 3B). Force-field calculations show that the experimentally observed nearest neighbor distance of $13.3 \AA$ agrees well with the theoretically determined distance of $13.0 \AA$. The interplay between (repulsive) steric hindrance and attractive interactions due to partial overlap between interdigitated aromatic units bestows a directionality to this type of aromatic binding motif, hence overcoming the limitation of non-directionality, rendering a rational design of supramolecular architectures possible. Other possible entities for aromatic binding motifs include different types of spiral aromatic units. [42]

While cHBC is achiral, the honeycomb networks on $\mathrm{Cu}(111)$ are chiral structures with the chirality of the networks defined by the coupling between nearest neighbors. ${ }^{[41]}$ The bay regions of $\mathrm{cHBC}$ can be regarded as helical tetrahelicene units. Each $\mathrm{cHBC}$ can then be considered as consisting of three fused M- or P-tetrahelicene units. Interdigitation is only possible by two tetrahelicene units of the same handedness. Therefore, honeycomb structures are enantiopure in their coupling motif which can be either based on $\mathrm{M}-\mathrm{M}$ or $\mathrm{P}-\mathrm{P}$ interdigitation. This represents a new class of surface-supported chiral objects, an area of research which has gained a lot of interest recently. ${ }^{[43]} \mathrm{A}$ further aspect of the cHBC honeycomb network is the chiral nature of its pores, which could be prone to enantioselectively host chiral guest molecules.

Due to the overlapping $\pi$-orbitals, the cHBC networks are expected to show a significant charge-carrier mobility, which would represent - to the best of our knowledge - the first truly 2D system for which this is the case. An AM1 calculation (Fig. $3 \mathrm{C})$ shows that the HOMO and LUMO levels of the cHBC dimer in the interdigitated geometry are split, with the splitting increasing if the molecules are brought closer together. Using Marcus theory, this splitting is found to be proportional to the electron/ hole mobility. ${ }^{[4]}$ In the present case, the HOMO splitting is larger, suggesting that the hole mobility exceeds the electron mobility, which is commonly observed for organic semiconductors. ${ }^{[45]}$

Besides their use in truly 2D supramolecular architectures, aromatic interactions have also proven efficient for the binding of fullerene $\mathrm{C}_{60}$ guest molecules to a surface-supported superlattice of corannulene hosts. ${ }^{[46]}$ Fig. 4 shows the ordering of $\mathrm{C}_{60}$ on a corannulene monolayer on $\mathrm{Cu}(110)$. As highlighted in Fig. 4B, each $\mathrm{C}_{60}$ guest sits on top of a corannulene host which is visualized by the model in Fig. 4C. Interestingly, variable-temperature STM studies reveal two distinctly different states of $\mathrm{C}_{60}$ on the corannulene host lattice, with
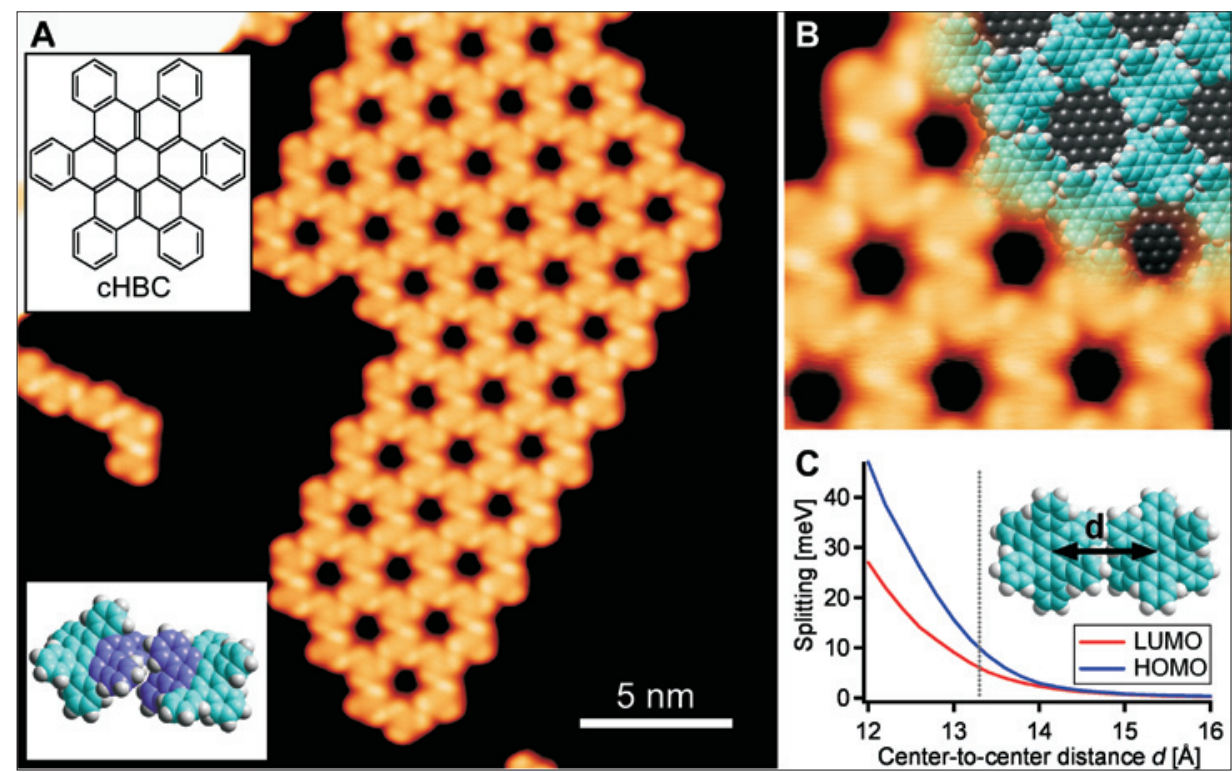

Fig. 3. (A) STM image of $\mathrm{CHBC}$ on $\mathrm{Cu}(111)$. The upper inset shows the chemical structure of cHBC. The lower inset shows two interdigitated nearest neighbours with the interdigitating tetrahelicene units being highlighted in violet. (B) High-resolution STM image of the honeycomb phase with superposed structural model. (C) Splitting of the HOMO/LUMO levels as computed with AM1 as a function of nearest neighbour distance in the self-complementary nearest neighbour recognition geometry. 


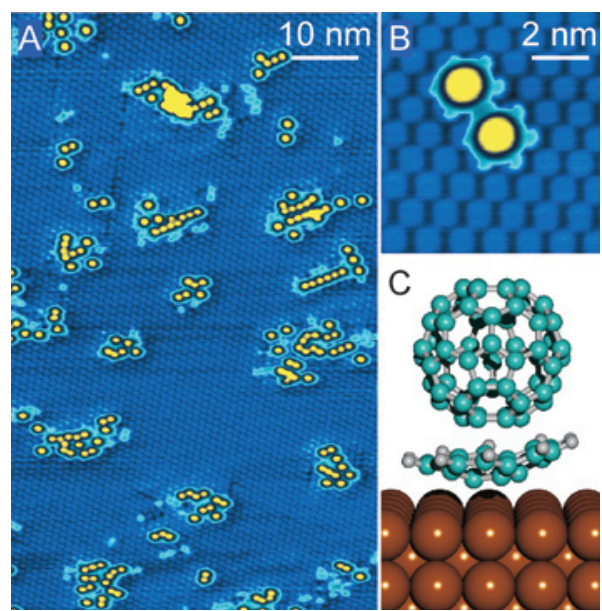

Fig. 4. Overview $(A)$ and high-resolution $(B)$ STM images of $\mathrm{C}_{60}$ guests on a corannulene host lattice supported on $\mathrm{Cu}(110)$. Contrast is applied such that $\mathrm{C}_{60}$ appears yellow while corannulene is dark blue. (C) Structural model of the corannulene- $\mathrm{C}_{60}$ host-guest complex on $\mathrm{Cu}(110)$

different binding energies and bowl-ball separations. This transition from a weakly bound precursor state to a strongly bound host-guest complex is found to be thermally activated, and has been shown to originate from a subtle interplay between homo- and heteromolecular interactions. ${ }^{[46]}$

\section{Nanoscale Phase Separation in Binary Molecular Systems}

When going from mono-molecular networks such as the ones presented above to multi-component supramolecular architectures, both homo- and hetero-molecular interactions have to be considered when designing (respectively understanding) a particular network. While the interaction with the substrate may influence the respective interactions due to charge transfer between substrate and adsorbate or due to substratemediated interactions, ${ }^{[47]}$ co-adsorption on coin metal surfaces often only leads to a change in absolute interaction energies while relative interaction energies - compared to gas-phase values - are approximately preserved for most systems. This allows - to a certain extent - for a rational design of $2 \mathrm{D}$ supramolecular networks. Co-adsorbates containing complementary functional units with hetero-molecular interactions dominating over their homo-molecular counterparts have successfully been used to produce 2D bi-component networks. ${ }^{[23,29]}$ First attempts towards three-component architectures have also been reported recently. ${ }^{[48]}$ The other extreme case, where homo-molecular interactions exceed hetero-molecular non-covalent bonds is of limited interest since this will simply lead to a phase separation of adsorbates. Astonishingly, there have been only very few reports about the intermediate region - where homo- and hetero-molecular interactions are of comparable strength, such that the thermodynamically most stable supramolecular structure will correspond to an optimized balance of both interactions.

We have investigated the self-assembled superstructures formed by the organic semiconductor 3,4,9,10-perylenetetracarboxylic-dianhydride (PTCDA) when coadsorbed with amine functionalized molecules 4,4'-diamino- $p$-terphenyl (DATP) and 2,4,6-tris(4-aminophenyl)-1,3,5-triazine (TAPT). Chemical structures are given in the Scheme. Fig. 5 illustrates the range of low-dimensional PTCDA structures that can be obtained by co-adsorption of DATP and TAPT on $\mathrm{Au}(111)$. A unifying feature of all PTCDA nanostructures is that they contain PTCDA-PTCDA interaction geometries that can also be found in the monolayer phases of PTCDA on $\mathrm{Au}(111){ }^{[49]}$
The amine-functionalized co-adsorbates hence only act as hydrogen-bonding spacers between pure phases of PTCDA with nanoscale dimensions.

Neither DATP or TAPT are able to form strong homo-molecular hydrogen bonds because of their lack of hydrogenbond acceptor units. Also, van-der-Waals interactions are small because of the limited contact surface areas due to the planar adsorption geometries. Homomolecular interactions between these two species can hence be neglected compared to hetero-molecular hydrogen bonds with PTCDA. PTCDA, in turn, can form strong multiple homo-molecular hydrogen bonds which are rather flexible in terms of intermolecular distance and relative molecular orientation, leading to a range of stable interaction geometries as confirmed by an extensive DFT analysis, ${ }^{[50]}$ which is also reflected in different types of PTCDA monolayer superstructures that have been observed.[51]

This example shows that by exploiting subtle energy balances between versatile homo- and heteromolecular interactions, highly ordered arrays of nanoscale phaseseparated structures can be grown via self-assembly. While a rational design of such structures may be difficult due to the manifold and complex interactions that are involved in final structure formation, the identification of suitable pairs of functional units - such as the amine-anhydride combination shown here - is possible by the use of combined experimental and theoretical studies.

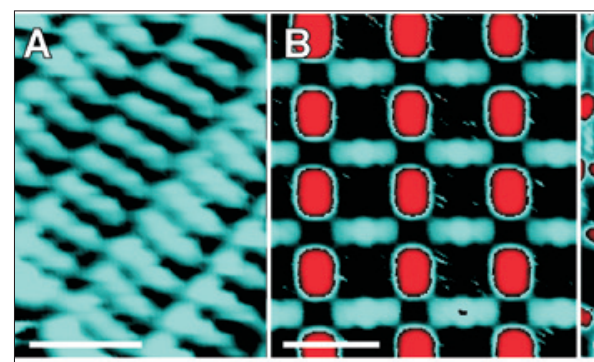

Spacers

OD PTCDA
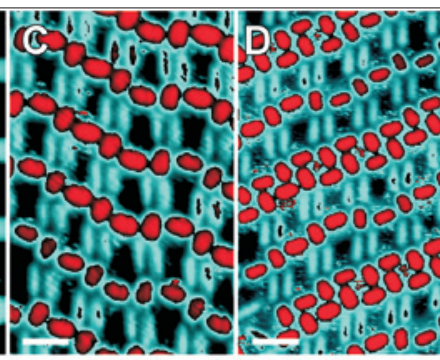

1D PTCDA

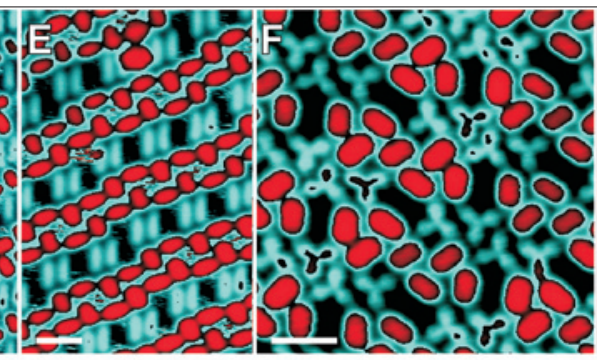

2D PTCDA

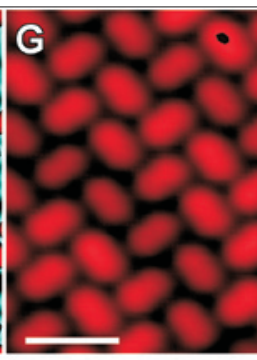

PTCDA

Fig. 5. Series of STM-images showing the range of PTCDA nanostructures that can be grown on Au(111) using hydrogen bonding spacers. Contrast has been applied such that spacer molecules appear in turquoise while PTCDA appears red. (A) Densely packed monolayer phase of DATP spacers. (B) Rectangular PTCDA-DATP network. (C-E) Linear PTCDA single (C), alternating single/double (D) and double ribbons (E) spaced by DATP pairs. (F) Array of PTCDA nanoislands consisting of 7 molecules spaced by TAPT. (G) Monolayer PTCDA herringbone phase. The scale bar is 2 nm in all images. 


\section{Surface Chemistry Approaches to Low-dimensional Polymeric Nanostructures}

Among the most promising potential applications of 2D supramolecular porous networks is their use as nanoscale templates for the creation of organic/inorganic nanostructures with dimensions well below those achievable by lithographic techniques. Furthermore, such networks can be tuned in both shape and size by chemical synthe$\operatorname{sis}^{[14]}$ and it has been shown that they allow for the templated growth of fullerene clusters ${ }^{[29]}$ or the trapping of large adsorbates. ${ }^{[52]}$ Recently it has also been shown that Co and Fe atoms selectively decorate a surface-supported metal-coordination network to form small clusters. ${ }^{[53]}$ However, routinely obtained networks based on non-covalent interactions such as hydrogen bonds, ${ }^{[29]}$ metal co-ordination ${ }^{[30]}$ or aromatic interactions ${ }^{[41]}$ do not generally meet the stringent criteria of thermal and chemical stability needed for their efficient use in the production of technologically relevant objects such as magnetic nanostructures or nanoscale sensor arrays. To overcome this limitation, there have recently been several attempts towards covalently bound surface-supported submonolayer thick polymeric structures. ${ }^{[54-57]}$ Successful proof-of-principle studies have exploited the oligomerization of $\mathrm{N}$ heterocyclic carbenes, imine-formation by condensation between aldehydes and amines, ${ }^{[56]}$ condensation of boronic acids, ${ }^{[57]}$ coupling of porphyrins through $\mathrm{C}-\mathrm{C}$ linkage via elimination of $\mathrm{Br}_{2}{ }^{[58]}$ and via a radical mechanism supported by a copper substrate. ${ }^{[59]}$

We have investigated the surface-confined condensation reaction between anhydrides and amines which leads to polyimides (Fig. 6A). ${ }^{60,61]}$ In solution and in thin films, the amic acid reaction intermediate is readily formed at room temperature, whereas on $\mathrm{Au}(111)$ structures ascribed to amic acid species (Fig 6B) can only be observed after annealing to above $\sim 470 \mathrm{~K} .{ }^{[61]}$ As outlined in the previous section, below this temperature, the two species form highly ordered nanostructures stabilized by hydrogen bonds. ${ }^{[50]}$ The restriction of the two reaction partners to two dimensions hence considerably raises the activation barrier for the first reaction step which is intuitively plausible since the nucleophilic backside attack involved in this step requires an offset between the reaction partners, which might only be reached at higher temperatures where vibrational amplitudes of either of the reactants orthogonal to the surface become significant. After annealing to above $\sim 570 \mathrm{~K}$, polyimide structures are formed. For the combination of DATP and PTCDA, linear strands which can be locally parallel (Fig. 6C,D) due to inter-chain hydrogen bonding are observed. Typical lengths of these strands are on the order of 5-10 molecules, which is considerably smaller than for polymeric structures formed in more 'traditional' chemical environments. This smaller size might be due to kinetic limitations during growth and/or to steric hindrance. Interestingly, the confinement of the imidization condensation reaction to the surface appears to increase the proportion of iso-imide reaction products, ${ }^{[61]}$ where the nitrogen has replaced the carbonyl oxygen instead of the bridging oxygen in the final product. This is tentatively ascribed to a lower strain energy of both the intermediate and final reaction products of the iso-imide in the constrained geometry on $\mathrm{Au}(111)$. ${ }^{61]}$

The overall topographic properties of the polymeric network can be influenced by changing the reaction partners. While the linear DATP leads to locally close-packed films of one-dimensionally interconnected oligomers (Fig. 6C and D), reacting PTCDA with the three-fold symmetric TAPT leads to extended 2D connectivity and a porous network (Fig. 6E and F) which might serve as a template for the growth of metal clusters. However, covalent networks with significant long-range order could not be obtained under the chosen surface-confined imidization conditions. This also applies to all other surface polymerization reactions reported so far. ${ }^{[55-59]}$ The formation of defect-free continuous polymer networks thus represents a major challenge in this new field. Further studies will have to explore the possibilities of topological polymerization reactions in preordered networks or self-repair mechanisms that allow to dissociate and reconnect a mislinked unit at the correct position.

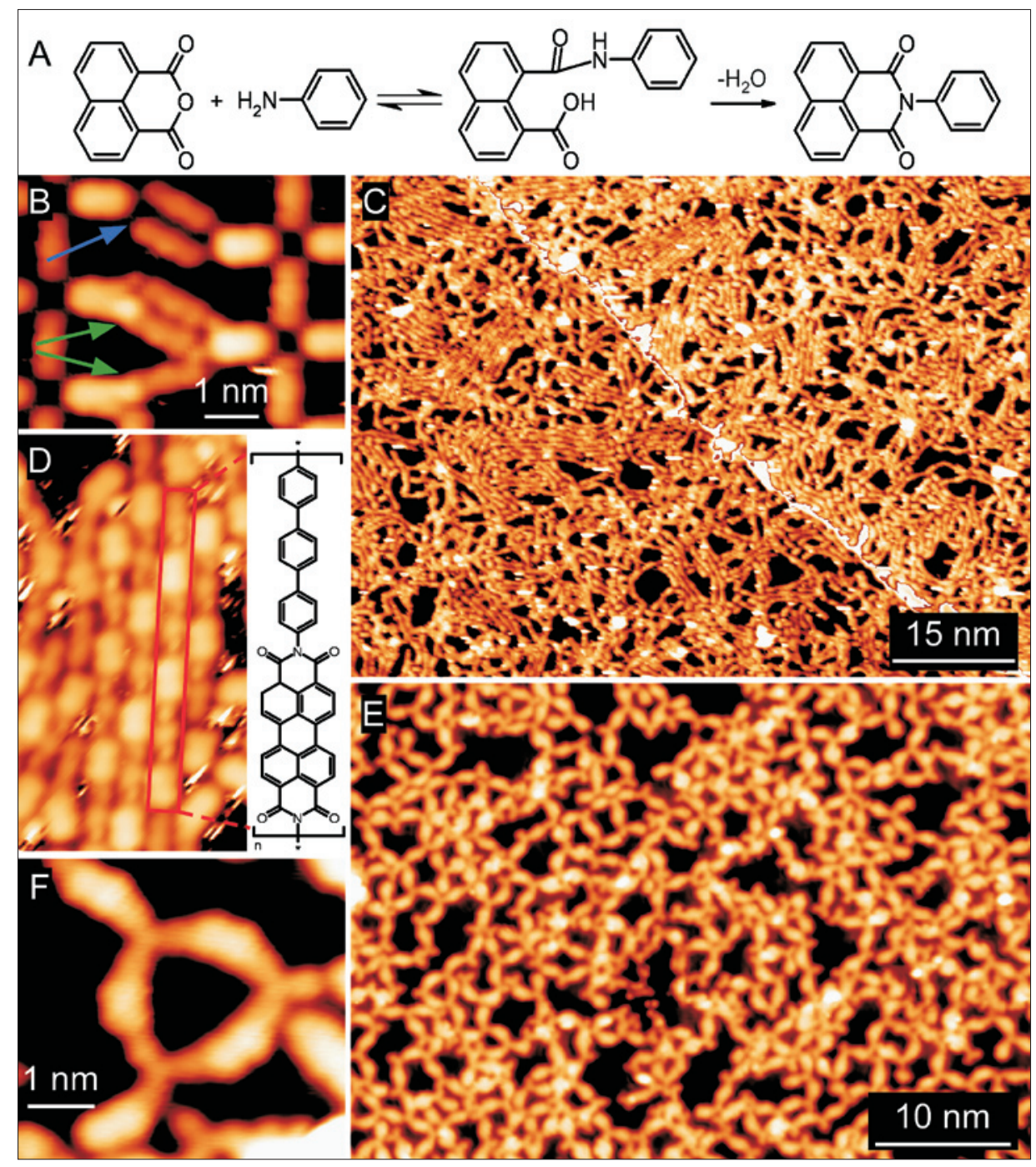

Fig. 6. (A) Imidization condensation reaction scheme. Anhydride and amine react to an amic acid reaction intermediate and finally to the imide by shedding water. (B) Amic acid reaction intermediates incorporated in non-reacted, hydrogen-bonded structures. Green arrows highlight protrusions centred at PTCDA-DATP junctions which are ascribed to amic acid while the blue arrow points to the corresponding hydrogen bonded junction where no protrusion is visible. (C) Large scale STM image of linear polyimide oligomers formed between DATP and PTCDA comprising locally aligned patches of co-parallel ribbons. (D) High-resolution STM image of coparallel PTCDA-DATP polyimide ribbons. (E) Large scale STM image of 2D interconnected porous polyimide network formed by fully reacted TAPT and PTCDA. (F) High resolution STM image of an individual pore from (E). 


\section{Conclusions}

The presented case studies illustrate the richness of surface chemistry and its relevance in the bottom-up fabrication of surface-supported organic nanostructures. As shown in this short review, a wealth of intriguing phenomena are observed at the interface between chemistry and physics, which can differ considerably from seemingly analogous processes within other fields at the core of either discipline. Understanding the interactions between large organic adsorbates and substrates and the resulting implications for self-assembly, reactivity and electronic properties remains challenging and requires both progress in theoretical modeling and the deduction of generally applicable concepts from extensive experimental studies.

While there is rapidly growing knowledge to rationally design supramolecular architectures on surfaces, future work will have to focus on increasing the stability and selectively functionalizing supramolecular networks to render them more suitable for possible applications. A key step in this respect will consist in moving towards more 'realistic' sample preparation conditions, such as pressures well above UHV conditions. Also, the creation of organic nanostructures on semiconductors, insulators or insulating thin films will become more important in this context. As a first step along these lines, we are currently investigating whether the concept of template-directed anchoring and growth can be extended to naturally templated insulating films such as for example ultrathin aluminum oxide on $\mathrm{Ni}_{3} \mathrm{Al}(111) \cdot{ }^{62]}$

Regarding the functionalization of supramolecular networks, first successful attempts towards functional pores have recently been reported, ${ }^{[63]}$ paving the way for future nanoscale sensor arrays. Aromatic coupling motifs ${ }^{[41]}$ are inherently functional since they combine geometrical organization with electronic coupling and might therefore be of particular interest in bestowing electronically relevant properties to a network. It will most probably also be necessary to combine different classes of non-covalent and covalent interactions to steer the selfassembly in order to obtain a desired functionality and topology. This may also require going beyond purely 2D systems towards rationally designed hierarchically assembled thin films. First attempts towards a stepwise self-assembly into the third dimension have been reported recently. ${ }^{[64]}$

\section{Acknowledgements}

Financial support from the Swiss National Science Foundation is gratefully acknowledged. We would like to thank Klaus Müllen, Colin Nuckolls and Neil R. Champness for providing the substances used in this work, and the groups of Daniele Passerone and Werner Hofer for continued theoretical support.

\section{Received: January 15, 2009}

[1] G. Ertl, Angew. Chem., Int. Ed. 2008, 47, 3524.

[2] Z. Ma, F. Zaera, Surf. Sci. Rep. 2006, 61, 229.

[3] F. Rosei, M. Schunack, Y. Naitoh, P. Jiang, A. Gourdon, E. Laegsgaard, I. Stensgaard, C. Joachim, F. Besenbacher, Prog. Surf. Sci. 2003, 71,95 .

[4] B. E. Bent, Chem. Rev. 1996, 96, 1361

[5] C. Joachim, J. K. Gimzewski, A. Aviram, Nature 2000, 408, 541.

[6] F. Chen, J. He, C. Nuckolls, T. Roberts, J. E. Klare, S. Lindsay, Nano Lett. 2005, 5, 503.

[7] A. S. Kumar, T. Ye, T. Takami, B. C. Yu, A. K. Flatt, J. M. Tour, P. S. Weiss, Nano Lett. 2008, 8 , 1644

[8] F. Jackel, M. D. Watson, K. Mullen, J. P. Rabe, Phys. Rev. Lett. 2004, 92

[9] J. Tang, E. P. De Poortere, J. E. Klare, C. Nuckolls, S. J. Wind, Microelectron. Eng. 2006, 83, 1706

[10] G. M. Whitesides, B. Grzybowski, Science 2002, 295, 2418.

[11] C. D. Dimitrakopoulos, P. R. L. Malenfant, $A d v$. Mater. 2002, 14, 99.

[12] H. Hoppe, N. S. Sariciftci, J. Mater. Res. 2004 19, 1924.

[13] A. P. Kulkarni, C. J. Tonzola, A. Babel, S. A. Jenekhe, Chem. Mat. 2004, 16, 4556.

[14] J. V. Barth, Аnпu. Rev. Phys. Chem. 2007, 58, 375.

[15] S. De Feyter, F. C. De Schryver, J. Phys. Chem. B 2005, 109, 4290.

[16] K. Kuhnke, K. Kern, J. Phys.-Condes. Matter 2003, 15, S3311.

[17] H. Brune, M. Giovannini, K. Bromann, K. Kern, Nature 1998, 394, 451

[18] N. Witkowski, Y. Borensztein, G. Baudot, V. Repain, Y. Girard, S. Rousset, Phys. Rev. B 2004, 70.

[19] H. Brune, C. Romainczyk, H. Roder, K. Kern, Nature 1994, 369, 469

[20] P. Ruffieux, K. Palotas, O. Groning, D. Wasserfallen, K. Mullen, W. A. Hofer, P. Groning, R. Fasel, J. Am. Chem. Soc. 2007, 129, 5007.

[21] M. Treier, P. Ruffieux, R. Schillinger, T. Greber, K. Mullen, R. Fasel, Surf. Sci. 2008, 602, L84.

[22] P. Ruffieux, O. Groning, R. Fasel, M. Kastler, D. Wasserfallen, K. Mullen, P. Groning, J. Phys. Chem. B 2006, 110, 11253 .

[23] M. E. Canas-Ventura, W. Xiao, D. Wasserfallen, K. Mullen, H. Brune, J. V. Barth, R. Fasel, Angew. Chem., Int. Ed. 2007, 46, 1814.

[24] W. D. Xiao, P. Ruffieux, K. Ait-Mansour, O. Groning, K. Palotas, W. A. Hofer, P. Groning, R. Fasel, J. Phys. Chem. B 2006, 110, 21394.

[25] K. Ait-Mansour, A. Buchsbaum, P. Ruffieux, M. Schmid, P. Groning, P. Varga, R. Fasel, O. Groning, Nano Lett. 2008, 8, 2035

[26] S. Berner, M. Corso, R. Widmer, O. Groening, R. Laskowski, P. Blaha, K. Schwarz, A. Goriachko, H. Over, S. Gsell, M. Schreck, H. Sachdev, T. Greber, J. Osterwalder, Angew. Chem., Int. Ed. 2007, 46, 5115

[27] D. Ecija, M. Trelka, C. Urban, P. de Mendoza, A Echavarren, R. Otero, J. M. Gallego, R. Miranda, Appl. Phys. Lett. 2008, 92, 3

[28] S. Clair, W. Pons, H. Brune, K. Kern, J. V. Barth, Angew. Chem., Int. Ed. 2005, 44, 7294.

[29] J. A. Theobald, N. S. Oxtoby, M. A. Phillips, N R. Champness, P. H. Beton, Nature 2003, 424, 1029 .

[30] U. Schickum, R. Decker, F. Klappenberger, G. Zoppellaro, S. Klyatskaya, M. Ruben, I. Silanes, A. Arnau, K. Kern, H. Brune, J. V. Barth, Nano Lett. 2007, 7, 3813

[31] S. Stepanow, M. Lingenfelder, A. Dmitriev, H. Spillmann, E. Delvigne, N. Lin, X. B. Deng, C. Z. Cai, J. V. Barth, K. Kern, Nat. Mater. 2004, 3 , 229.
[32] A. Langner, S. L. Tait, N. Lin, R. Chandrasekar, M. Ruben, K. Kern, Angew. Chem., Int. Ed. 2008, 47,8835

[33] G. R. Desiraju, Angew. Chem., Int. Ed. 1995, 34 , 2311.

[34] C. A. Hunter, K. R. Lawson, J. Perkins, C. J. Urch, J. Chem. Soc.-Perkin Trans. 2001, 2, 651.

[35] G. R. Desiraju, Acc. Chem. Res. 2002, 35, 565.

[36] J. S. Wu, Curr. Org. Chem. 2007, 11, 1220.

[37] A. R. Murphy, J. M. J. Frechet, Chem. Rev. 2007, 107, 1066.

[38] V. A. Langlais, Y. Gauthier, H. Belkhir, O. Maresca, Phys. Rev. B 2005, 72

[39] M. C. Blum, E. Cavar, M. Pivetta, F. Patthey, W. D. Schneider, Angew. Chem., Int. Ed. 2005, 44, 5334.

[40] S. X. Xiao, M. Myers, Q. Miao, S. Sanaur, K. L. Pang, M. L. Steigerwald, C. Nuckolls, Angew. Chem., Int. Ed. 2005, 44, 7390.

[41] M. Treier, P. Ruffieux, P. Groning, S. X. Xiao, C. Nuckolls, R. Fasel, Chem. Commun. 2008, 4555.

[42] C. Schmuck, Angew. Chem., Int. Ed. 2003, 42 2448

[43] K. H. Ernst, Curr. Opin. Colloid Interface Sci. 2008, 13,54 .

[44] W. Q. Deng, W. A. Goddard, J. Phys. Chem. B 2004, 108, 8614

[45] J. L. Bredas, J. P. Calbert, D. A. da Silva, J. Cornil, Proc. Natl. Acad. Sci. U. S. A. 2002, 99, 5804.

[46] W. Xiao, D. Passerone, P. Ruffieux, K. Ait Mansour, O. Groning, E. Tosatti, J. S. Siegel, R. Fasel, J. Am. Chem. Soc. 2008, 130, 4767.

[47] S. Lukas, G. Witte, C. Woll, Phys. Rev. Lett. 2002 88

[48] A. Llanes-Pallas, M. Matena, T. Jung, M. Prato, M. Stohr, D. Bonifazi, Angew. Chem., Int. Ed. 2008, 47, 7726

[49] S. Mannsfeld, M. Toerker, T. Schmitz-Hübsch, F. Sellam, T. Fritz, K. Leo, Org. Electron. 2001, 2 , 121

[50] M. Treier, M.-T. Nguyen, N. V. Richardson, C. Pignedoli, D. Passerone, R. Fasel, Nano Lett. 2009, 9, 126.

[51] F. S. Tautz, Prog. Surf. Sci. 2007, 82, 479.

[52] A. R. Bruno Schmaltz, Hans Joachim Räder, Wojciech Pisula, Klaus Müllen,, Angew. Chem. Int., Ed. 2009, 48, 720 .

[53] R. Decker, U. Schlickum, F. Klappenberger, G. Zoppellaro, S. Klyatskaya, M. Ruben, J. V. Barth H. Brune, Appl. Phys. Lett. 2008, 93, 243102.

[54] A. Gourdon, Angew. Chem., Int. Ed. 2008, 47, 6950.

[55] M. Matena, T. Riehm, M. Stohr, T. A. Jung, L. H Gade, Angew. Chem., Int. Ed. 2008, 47, 2414

[56] S. Weigelt, C. Busse, C. Bombis, M. M. Knudsen, K. V. Gothelf, E. Laegsgaard, F. Besenbacher, T. R. Linderoth, Angew. Chem., Int. Ed. 2008, 47, 4406.

[57] N. A. A. Zwaneveld, R. Pawlak, M. Abel, D. Catalin, D. Gigmes, D. Bertin, L. Porte, J. Am Chem. Soc. 2008, 130, 6678.

[58] L. Grill, M. Dyer, L. Lafferentz, M. Persson, M V. Peters, S. Hecht, Nat. Nanotechnol. 2007, 2 687.

[59] M. I. Veld, P. Iavicoli, S. Haq, D. B. Amabilino, R. Raval, Chem. Commun. 2008, 1536.

[60] M. Treier, N. V. Richardson, R. Fasel, J. Am. Chem. Soc. 2008, 130, 14054.

[61] M. Treier, R. Fasel, N. R. Champness, S. Argent, N. V. Richardson, Phys. Chem. Chem. Phys. 2009, 11, 1209

[62] S. Degen, A. Krupski, M. Kraj, A. Langner, C. Becker, M. Sokolowski, K. Wandelt, Surf. Sci. 2005, 576, L57.

[63] L. M. A. Perdigao, A. Saywell, G. N. Fontes, P A. Staniec, G. Goretzki, A. G. Phillips, N. R Champness, P. H. Beton, Chem.-Eur. J. 2008, 14 , 7600.

[64] M. Altman, O. Zenkina, G. Evmenenko, P. Dutta, M. E. van der Boom, J. Am. Chem. Soc. 2008 , 130, 5040 . 